\title{
On the Ability of Humans to Apply Controlled Forces to Admittance-Type Devices
}

\author{
Manikantan Nambi *, William R. Provancher and Jake J. Abbott \\ Department of Mechanical Engineering, University of Utah, Salt Lake City, UT 84112, USA
}

Received 11 May 2010; accepted 6 November 2010

\begin{abstract}
Human-robot collaborative systems have the potential to dramatically change many aspects of surgery, manufacturing, hazardous-material handling, and other dextrous tasks. We are particularly interested in precise manipulation tasks, which are typically performed under an admittance-control regime, where the controlled velocity of a non-backdrivable robot is proportional to the sensed user-applied force. During fast movements, there is a noticeable degradation in control precision and prior results have indicated that system velocity, and not system admittance, is the factor that is most correlated with force control precision. In this paper, we report evidence that system admittance is more important than velocity in determining the user's ability to control applied force and that both factors are less important than the force level itself, and we provide an explanation as to why prior results might have indicated otherwise. We find the conditions under which human force control performance is best when operating under admittance control. We also report the conditions under which human force control on a moving admittance-type device is indistinguishable from isometric force control, which can be used to design better device controllers.
\end{abstract}

(c) Koninklijke Brill NV, Leiden and The Robotics Society of Japan, 2011

\section{Keywords}

Human force control, admittance control, proportional-velocity control, haptics

\section{Introduction}

Human-robot collaborative systems can take the form of teleoperation systems, where movement of a master manipulandum is coupled to movement of a remote slave manipulator, or they can take the form of cooperative systems, where the human directly interacts with a robotic manipulator. For cooperative tasks requiring the greatest degree of precision, admittance-type robots are often used, which are devices that contain a great deal of gearing and inertia such that they appear nonbackdrivable to a human, as well as to many disturbances from the environment [1].

\footnotetext{
* To whom correspondence should be addressed. E-mail: m.nambi@utah.edu
} 
It is challenging to stably implement precise position control with impedance-type devices (i.e., backdrivable, low inertia, low friction) because impedance-type control systems do not sufficiently penalize small disturbances from the environment and from human tremor. Admittance-type robots are controlled using admittance control: a human interacts directly with a force sensor mounted to the robot and the robot is computer-controlled to move in response to the applied force. The most common and simplest type of admittance control is proportional-velocity control, where the admittance of the system reduces to a simple gain $k$, making the velocity of the robot $V$ linearly proportional to the applied force $F$ :

$$
V=k F \text {. }
$$

If perfectly implemented, the control law (1) behaves like a massless viscous damper, with damping inversely proportional to the gain $k$. It also behaves similarly to a 'programmable damper' implemented on an impedance-type haptic device; however, it is possible to stably implement much higher levels of damping with an admittance-type device, including perfectly rigid systems. An example of an existing system that uses the control law (1) is the Johns Hopkins University SteadyHand Robot [2,3], which is used for tasks such as the insertion of a tiny needle into a retinal vein that is smaller than the size of the tremor in the surgeon's hand. Another example of a system that uses the control law (1) is the University of Utah Active Handrest [4], which is a handrest for manual tasks that can be repositioned intuitively by the user.

Precise tasks are typically performed at low velocities and it seems somewhat obvious that positioning precision will be maximized in the limit as the admittance gain $k$ is reduced to zero, with positioning precision ultimately bounded by the position-sensing resolution. However, a very low value of $k$ will result in a system that is too sluggish, and possibly too fatiguing, to be useful for any real task. We would like the user to be able to move as fast as possible and still maintain maximum precision, but we find that as the admittance gain $k$ is increased, the system eventually begins to feel uncomfortable and somewhat out of control. We find that it is difficult for a user to control the level of applied force on the admittance-type device when the device is too responsive. We believe that human users are most confident interacting with devices that are somewhat dissipative (as opposed to only passive), and that there will exist a range of forces and velocities within which humans optimally interact with devices - considering human ability to control force, confidence in interaction (e.g., humans may feel most comfortable interacting with devices that feel natural) and fatigue due to excessive force application. Additionally, robust stability analysis of haptic systems typically involves conservative passivity-based techniques [1], due to the complex exchange of information and energy between the human user and the robotic device. If conditions could be found in which we could guarantee that the human user was unaffected by the motion of the robotic device, then the human could be treated as an exogenous system input, which would allow less-conservative stability conditions to be 
applied to the robot's control system. Thus, a better understanding of human force control during admittance control would help us design better systems for precise and efficient manipulation.

The purpose of this research is 2-fold: (i) to quantify the effects of velocity $(V)$, admittance gain $(k)$ and force level $(F)$ on human force control ability with visual and haptic feedback, and (ii) to determine under which conditions human force control of an admittance-type device is indistinguishable from isometric force control (i.e., force applied to a stationary sensor), implying that the human's applied force is not affected by the movement of the device. Although we are explicitly considering the ability to precisely control force in this work, we are ultimately interested in systems in which we are precisely controlling position under direct visual feedback using the admittance-control law (1). For an admittance-type device operating at low speeds, the user is always applying force in the direction of motion, so operation in three-dimensional space can be considered as applying force on a 1-d.o.f. system at each instant of time. Thus, it is our assertion that our study, which is conducted on such a 1-d.o.f. system, is extensible to spatial motion.

Several studies have been conducted on human ability to apply force on a static object. A mean absolute error of 11-15\% of the target force value (computed as (7) herein) was found by Srinivasan and Chen [5] when applying a constant force on a stationary target in the range of $0.25-1.25 \mathrm{~N}$ with no visual feedback. In the same experiment they found that the error reduced significantly and remained approximately constant at $0.039 \mathrm{~N}$ when visual feedback was provided (3-16\%). Allin et al. [6] found the just noticeable difference (JND) for force to be at $10 \%$ for the index finger for a base force of $2.25 \mathrm{~N}$. Visual feedback was provided to the user in this experiment. A few studies have been conducted on human force control on moving objects. Lederman et al. [7] studied the force variability in the normal direction with the subject moving their hand at different velocities ( 20 and $222 \mathrm{~mm} / \mathrm{s}$ ) in the tangential direction and under different force levels (user-defined 'low force' and 'medium force'). They found the end-effector, force level, and velocity of the device to have a statistically significant effect on the mean force values.

In the study most closely related to ours, Wu et al. [8] (prior work of one of the current authors) studied the effect of velocity and admittance gain on subjects applying force on a moving robotic device. In two separate experiments, the robotic device was controlled under admittance control and under velocity control (i.e., independent of the applied force), respectively. They determined that the velocity $(V)$, and not admittance gain $(k)$, directly affects force control ability. However, the conclusion of Ref. [8] runs counter to more recent anecdotal experiences in the authors' labs, motivating a reconsideration of the findings. In this paper, we report evidence that, at least for the range of velocities and admittance gains considered herein, the fundamental conclusion of Ref. [8] does not appear to be correct for systems running under admittance control and that the admittance gain $(k)$ is in fact the dominant factor in a human's ability to precisely control the force applied to an admittance-type device. A number of choices made during the design of 
the experiment in Ref. [8] could have contributed to its conclusion. First, 50\% of the data obtained were under velocity control, which may not be representative of performance under proportional-velocity control. Second, in the experiments, the controllers were turned on after the human subject reached a desired target force, creating large accelerations at the beginning of each trial that may have affected the results. With real admittance systems, the velocity would naturally ramp up from rest as the human makes contact and begins to apply force. In addition, the experiments in Ref. [8] were conducted with only haptic feedback, requiring the subject to remember a target force value. It is not clear if the discrete event of turning the controller on and jerking up to speed affected the subjects' ability to remember the target force. In real-life systems, some method of visual feedback conveys information about the amount of applied force, typically the motion of the device. Research by Jones [9] indicates that the coefficient of variance for finger and elbow forces to be much lower with both visual and haptic feedback (4\%) than when only haptic feedback (12\%) is provided. Visual feedback in Ref. [9] was in the form of three vertical boxes on a computer screen. The center box was highlighted if the force applied by the user was within the bounds of the target box, the upper box was highlighted if force less than the target was applied, and the lower box was highlighted if force higher than the target force was applied.

The outline of the remainder of this paper is as follows. In Section 2, we describe the design of our experiment. In Section 3, we include the results of our experiment, and provide analysis. In Section 4, we provide additional discussion about the implications of the experimental results. We summarize our findings in Section 5.

\section{Methods}

\subsection{Hardware}

A 1-d.o.f. robotic device is used to perform the experiment (Fig. 1a). It consists of a lead-screw-driven linear stage (Servo Systems MLPS-4-10) driven by a DC motor (Servo Systems 23SMDC-LCSS). Position feedback is given by an optical encoder mounted on the motor shaft. The lead screw has a pitch of $12.7 \mathrm{~mm}$ and the encoder resolution is 4000 counts/rev after quadrature, which translates to a linear resolution of $3 \mu \mathrm{m}$ for the device. A cantilever-type force sensor was developed for the device. The force sensor has a sensitivity of $0.7 \mathrm{mN}$ per bit (noise below $0.01 \mathrm{~N}$ ) and is mounted on the linear stage using a rigid rod. A Sensoray 626 DAQ card is used for data acquisition. It has a 16-bit ADC that is used to read force data and a 14-bit DAC which is used to command voltages to the current amplifier (Advanced Motion Control 12A8), powered by a 24-V linear power supply, which is used to drive the motor. The voltage-to-current gain of the amplifier is $0.25 \mathrm{~A} / \mathrm{V}$. The software for the device was developed in C++ using the CHAI 3D library [10]. Visual feedback is provided to the subject on a $0.5-\mathrm{m}$ computer screen that was positioned $0.7 \mathrm{~m}$ from the user. The force readings are sampled at $1 \mathrm{kHz}$ and graphics are displayed at $33 \mathrm{~Hz}$. 


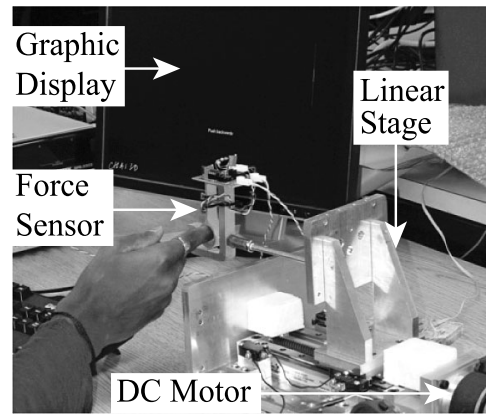

(a)

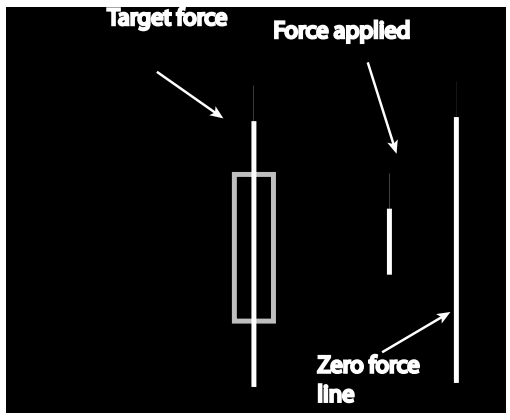

(b)

Figure 1. (a) Experimental setup with a subject applying force on a 1-d.o.f. robotic device. (b) Display on computer screen during the experiment showing the force applied by the subject, the target force and the green box with width set at $10 \%$ of target force.

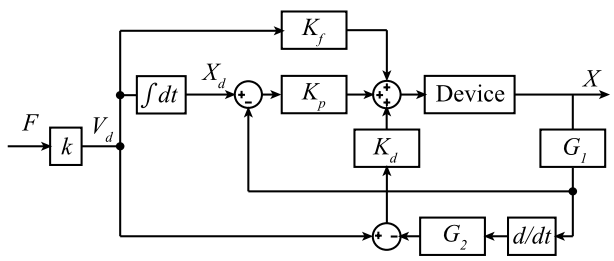

Figure 2. Control system.

\subsection{Control System}

A PD-plus-feedforward controller has been implemented for the device (Fig. 2). Unit-DC-gain digital low-pass filters $\mathrm{G}_{1}$ and $\mathrm{G}_{2}$ with time constants $\tau_{1}=0.001 \mathrm{~s}$ and $\tau_{2}=0.0005 \mathrm{~s}$ are used to reduce quantization error and differentiation noise. The proportional gain $K_{\mathrm{p}}$ is set at $30 \mathrm{~V} / \mathrm{mm}$ and the derivative gain $K_{\mathrm{d}}$ is set at $0.1(\mathrm{~V} \cdot \mathrm{s}) / \mathrm{mm}$ for the majority of the experiments. The proportional gain $K_{\mathrm{p}}$ is increased to $60 \mathrm{~V} / \mathrm{mm}$ for low velocities of 0.1 and $0.2 \mathrm{~mm} / \mathrm{s}$ to improve tracking. These gain values were empirically found to give the minimal tracking error for sinusoidal position trajectories. The controller gains are decided by the target velocity in each trial, which is constant for a given trial. Hence, the controller gains are fixed for each trial. The feedforward model for the device was experimentally derived and is given by $K_{\mathrm{f}}=0.06 V_{\mathrm{d}}+2.2\left(1-\mathrm{e}^{-3.3 V_{\mathrm{d}}}\right) \mathrm{V} \cdot \mathrm{s} / \mathrm{mm}$, where $V_{\mathrm{d}}$ is the desired velocity. This feedforward model is a smooth function that approximates Coulomb-plus-viscous friction. The inputs for the system are calculated as:

$$
\begin{gathered}
V_{\mathrm{d}}(n)=k F(n) \\
X_{\mathrm{d}}(n+1)=X_{\mathrm{d}}(n)+V_{\mathrm{d}}(n) \Delta t .
\end{gathered}
$$

$F(n)$ is the force applied by the user at sample $n . X_{\mathrm{d}}(n)$ is the desired position of the device at sample $n$, which is found by numerically integrating the desired velocity. 

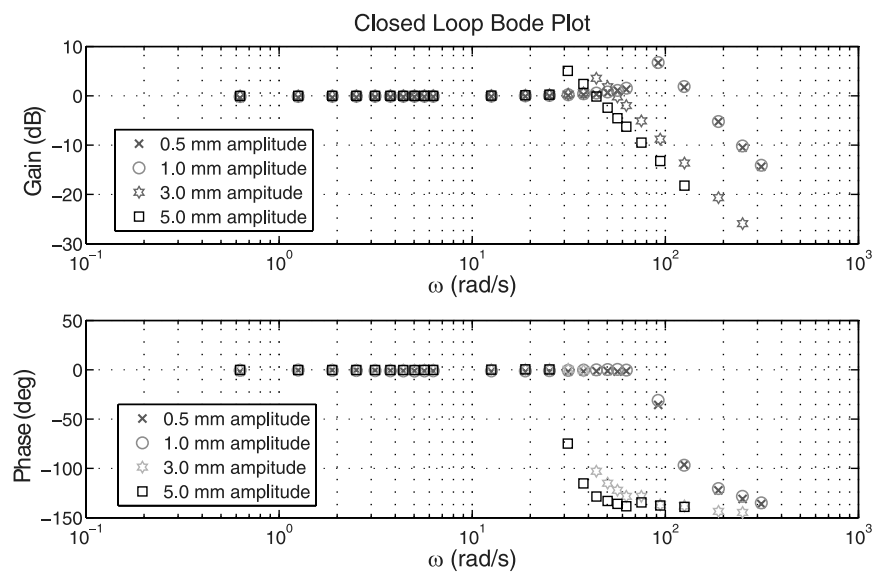

Figure 3. Experimental frequency-response plot for the closed-loop system for sinusoidal trajectories in $X_{\mathrm{d}}(t)$. For this device characterization experiment, $X_{\mathrm{d}}(n)$ is a sampled version of $X_{\mathrm{d}}(t)$ and $V_{\mathrm{d}}(n)$ is a sampled version of $V_{\mathrm{d}}(t)$, which is the time derivative of $X_{\mathrm{d}}(t)$. No human is in contact with the device.

$\Delta t$ is the sampling time of the control system $(1 \mathrm{~ms})$. The controlled device is capable of tracking signals at frequencies below $7 \mathrm{~Hz}(44 \mathrm{rad} / \mathrm{s})$, which is sufficient for signals of interest here (Fig. 3).

\subsection{Verification of Prior Work}

Using our device and a single human subject, we recreated the experiment performed in $\mathrm{Wu}$ et al. [8] to determine if the results were repeatable using our device. The subject had to apply force on a 1-d.o.f. force sensor (Fig. 1a) similar to the one used in Ref. [8]. Two experiments, one balanced in force $(F)$ and admittance gain $(k)$ and the other balanced in force $(F)$ and velocity $(V)$, were performed. Our analysis of variance (ANOVA) showed that both $k$ and $F$ have an effect on the metric (standard deviation of force for the second half of the trial when the device is moving, divided by the standard deviation of force for the first half of the trial when the device is stationary) in the experiment balanced in $k$ and $F$. For the experiment balanced in $V$ and $F$, only $V$ has an effect on the metric. These results are consistent with those obtained in Ref. [8], indicating that it is unlikely that the data collection or analysis in Ref. [8] were problematic. Rather, the reasons outlined in Section 1 are the likely causes for their conclusions.

\subsection{Experimental Design}

Our goal is to conduct experiments that give information specifically about human force control under the control law (1), rather than about human force control on moving objects in general. An experiment balanced in velocity $(V)$ and admittance gain $(k)$ was designed to test the effect of each factor on human force control precision. Specifically, we are interested in determining when force control ability is affected by the movement of the device, as compared to force control on a stationary 
Table 1.

Force $(\mathrm{N})$ values for different values of $V(\mathrm{~mm} / \mathrm{s})$ and $k(\mathrm{~mm} /(\mathrm{N} \cdot \mathrm{s}))$

\begin{tabular}{|c|c|c|c|c|c|c|c|c|c|c|c|}
\hline \multirow[t]{2}{*}{$V$} & \multicolumn{5}{|c|}{ (a) High-speed experiment $k$} & \multirow[t]{2}{*}{$V$} & \multicolumn{5}{|c|}{ (b) Low-speed experiment $k$} \\
\hline & 0.5 & 0.9 & 1.6 & 2.8 & 5.0 & & 0.1 & 0.18 & 0.32 & 0.56 & 1.0 \\
\hline 0.5 & 1 & 0.55 & 0.31 & 0.18 & 0.1 & 0.1 & 1 & 0.55 & 0.31 & 0.18 & 0.1 \\
\hline 0.9 & 1.8 & 1 & 0.56 & 0.32 & 0.18 & 0.18 & 1.8 & 1 & 0.56 & 0.32 & 0.18 \\
\hline 1.6 & 3.2 & 1.78 & 1 & 0.57 & 0.32 & 0.32 & 3.2 & 1.78 & 1 & 0.57 & 0.32 \\
\hline 2.8 & 5.6 & 3.11 & 1.75 & 1 & 0.56 & 0.56 & 5.6 & 3.11 & 1.75 & 1 & 0.56 \\
\hline 5.0 & 10 & 5.56 & 3.12 & 1.79 & 1 & 1.0 & 10 & 5.56 & 3.12 & 1.79 & 1 \\
\hline
\end{tabular}

device (isometric). The experiment was performed by nine right-handed subjects using their right index fingers. The subjects were all male, with ages ranging from 22 to 37. Only one of the subjects had prior experience with haptic interfaces. The experiment has Institutional Review Board approval. Results from pilot studies showed that $V$ and $k$ at which human force control precision is similar to the stationary case occurs for very slow moving systems. Also, limitations in the force sensor restrict the range of values of $V$ and $k$ that can be used. Therefore, in a first experiment, subjects were tested for target velocity values of $V=0.5,0.9,1.6,2.8$ and $5.0 \mathrm{~mm} / \mathrm{s}$ and admittance gains of $k=0.5,0.9,1.6,2.8$ and $5.0 \mathrm{~mm} /(\mathrm{N} \cdot \mathrm{s})$. A second experiment was performed with the values of $V=0.1,0.18,0.32,0.56$ and $1.0 \mathrm{~mm} / \mathrm{s}$ and admittance gains of $k=0.1,0.18,0.32,0.56$ and $1.0 \mathrm{~mm} /(\mathrm{N} \cdot \mathrm{s})$. The target force value to be applied by the subject was calculated using (1). Table 1 shows the target force level for each of the commanded admittance gains to achieve the desired velocity values. As $V$ and $k$ values have been equally scaled down by a factor of 5 for the low-speed experiment, the target force values in Table 1a and $\mathrm{b}$ are the same. It can bee seen from Table 1 that each combination of $V$ and $k$ has a different target force value associated with it.

For each different force value, trials with $k=0$ (isometric) are also conducted. These values are shuffled to generate a random sequence called a block. Eight such blocks with randomly distributed combinations of $V$ and $k$ are generated for each subject. Values of target force and admittance gains in a trial are selected according to these randomly generated blocks for each subject. The purpose of the blocks is to distribute the eight trials of each combination throughout the total duration of the experiment, in order to mitigate effects of learning and fatigue.

During the experiments, subjects are instructed to apply force on the force sensor using their right index finger. Their arm is placed perpendicular to the direction of motion of the device with their elbow supported on the table as shown in Fig. 1. Subjects applied force to the left, which is also the direction of motion. Visual feedback is provided on a computer screen in the form of a vertical grey line at the center of the screen indicating the target force to be achieved by the subject (see Fig. 1b). This grey line is surrounded by a green box whose width is set at $10 \%$ 


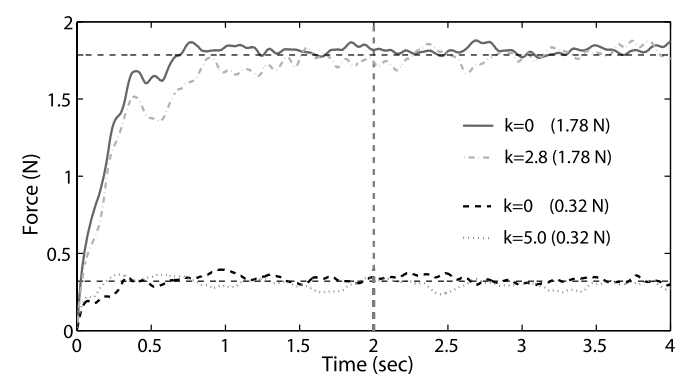

Figure 4. Typical force-versus-time data for stationary $(k=0)$ and moving systems. Here, a $0.32 \mathrm{~N}$ force is applied by a subject with $k=0$ and 5.0 , and a $1.78 \mathrm{~N}$ force is applied by a subject with $k=0$ and 2.8. Horizontal lines indicate the target forces. Only the final $2 \mathrm{~s}$ of data are used during analysis.

of the target force value. The width of the green box is $15 \mathrm{~mm}$ and the screen is $70 \mathrm{~cm}$ from the subject. The force applied by the subject is displayed by a white vertical line that moves from right to left when force is applied. The force display is scaled such that the center of the screen corresponds to the target force, the right edge corresponds to no force and the left edge corresponds to twice the target force. As the target force is always located at the center of the screen, a subject has no indication at the beginning of a trial as to what the target force is and must use the movement of the white vertical line accompanying their applied force in order to determine the correct force. Subjects are instructed to match the target force and keep the applied force within the green box to the best of their ability. Each trial lasts for $4 \mathrm{~s}$. The device begins moving at the instant the subject begins to apply force, based on (1). However, only the last $2 \mathrm{~s}$ of data are analyzed to remove the effects of the rise time required to achieve the target force, which is also present in the isometric case. Figure 4 shows typical force-versus-time responses for a subject pushing at two target forces, each at two different values of $k$, indicating how the mean force level applied by the subject changes as a function of movement of the device. White noise is played through headphones during the experiment to remove auditory cues. The device is hidden from the subjects by a curtain through which subjects reach their hand. Subjects could pause the experiment to rest at any time. The experiment took $50 \mathrm{~min}$, on average, to complete. Before the experiment, each subject was allowed to interact with the device and experience different force levels and admittance gains.

\section{Results}

The second, low-speed experiment was performed to find threshold values for $V$ and $k$ under which human force control precision is unaffected by movement. This second experiment was conducted after the discovery that the first experiment would not be sufficient to answer our questions of interest. The results of the first experiment (high-speed) are included as the Appendix for the interested reader. The remainder of this section will focus entirely on the low-speed experiment. The 


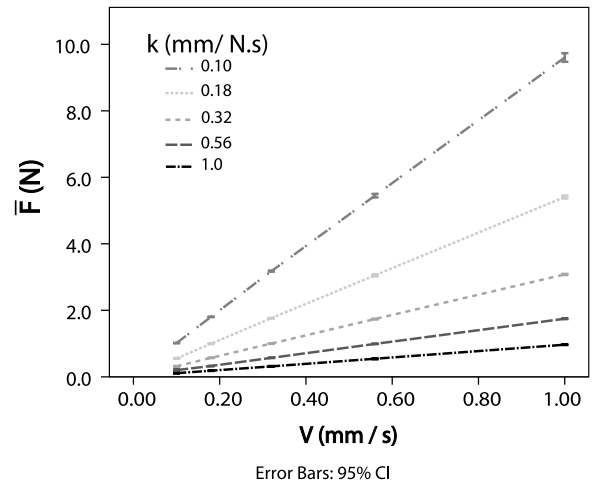

(a)

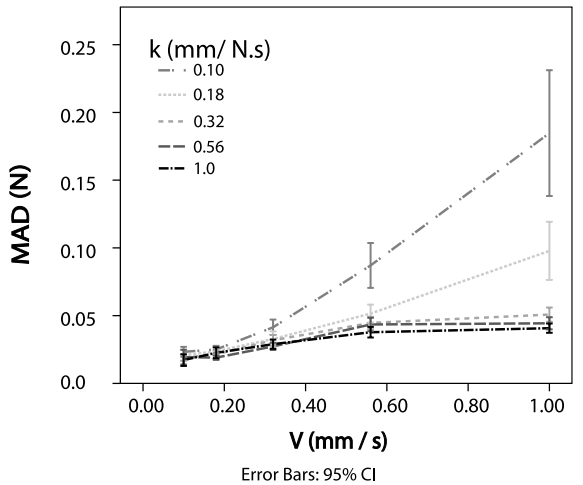

(b)

Figure 5. (a) Mean and (b) $M A D$ in force across all subjects and trials for different levels of $V$ and $k$.

data from the high-speed experiment were used in previously published work [11], where different metrics were used to study the effect of $V$ and $k$ on human force control precision. The metrics used in Ref. [11] were biased (i.e., not Gaussian), so the data has been reanalyzed with new metrics in this paper, described below.

$\bar{F}$ is the mean of the user's applied force (for the last $2 \mathrm{~s}$ of a given trial), which may differ from the target force. The mean force applied across subjects and trials is shown in Fig. 5a. Figure 5b shows the mean absolute deviation (MAD) in force, which is defined by:

$$
M A D=\frac{\sum\left|F_{x}-\bar{F}\right|}{N},
$$

where $F_{x}$ is the force value at each sampling instant (recorded at $1 \mathrm{kHz}$ ) and $N$ is the number of samples used (again, for the last $2 \mathrm{~s}$ of a given trial). $M A D$ is a measure of the subjects tremor around some mean value. The variance in both mean applied force and $M A D$ increase with increasing target force, which has been previously observed in the literature [9]. Figure 5 shows the combined effect of $V, k$ and $F$ on $\bar{F}$ and $M A D$ for all subjects across all trials. It is clear that the variance in mean force is quite small compared to the variance in $M A D$; this is an indication that visual feedback of force allows the subjects to accurately apply the target force, but the tremor of the subjects around that average (i.e., precision) is not eliminated by having visual feedback. We would like to isolate and study the effect of $V$ and $k$ on human force control ability. However, the effect of $V$ and $k$ on mean force and MAD are small compared to the effect of the target force level itself. Hence, the metrics used in the study were designed so as to remove the effect of force level on the force applied by the subject. We have used four metrics to compare force control ability. Two metrics measure the error in mean force, and the other two measure deviations in the applied force to capture human tremor and lack of precision around a mean force.

The first metric to measure error in mean force is the normalized error in mean 
force given by:

$$
E_{\mathrm{t}}=\frac{\bar{F}-F_{\mathrm{t}}}{F_{\mathrm{t}}},
$$

where $F_{\mathrm{t}}$ is the target force to be applied by the subject. The second metric to measure error in mean force is the normalized difference in mean force between a given trial and the isometric static population mean at a given target force level:

$$
E_{\mathrm{s}}=\frac{\bar{F}-\bar{F}_{\mathrm{ip}}}{\bar{F}_{\mathrm{ip}}},
$$

where $\bar{F}_{\text {ip }}$ is the isometric static population mean at a given target force level, which is the mean force applied in the isometric case (i.e., $k=0$ ), averaged across all subjects and trials for a given target force. Nine subjects were used in the experiment with eight repetitions for each force level in the isometric case. Hence, $\bar{F}_{\text {ip }}$ is a mean of 72 values, indicating what forces subjects actually apply, on average, when asked to apply a given force isometrically. $E_{\mathrm{t}}$ is a measure of the subject's ability to maintain a constant target force as compared to the target force and $E_{\mathrm{s}}$ is a measure of the subject's ability to maintain a constant target force as compared to the population's ability to maintain the same target force isometrically.

The first metric used to measure deviations in applied force is the normalized mean absolute deviation:

$$
M A D_{\mathrm{t}}=\frac{M A D}{F_{\mathrm{t}}},
$$

where $M A D$ is the mean absolute deviation for a trial, defined in (4). $M A D_{\mathrm{t}}$ is a particularly relevant metric for analysis of admittance-type systems under the control law (1): we have choice over the admittance gain $k$ to relate velocity to the commanded input force, so $M A D_{\mathrm{t}}$ is effectively a measure of the normalized input noise that will be observed in the system. The second metric to measure deviations in applied force is the normalized difference in $M A D$ between a given trial and the isometric static population mean of $M A D$ :

$$
M A D_{\mathrm{s}}=\frac{M A D-\overline{M A D}_{\mathrm{ip}}}{\overline{M A D}_{\mathrm{ip}}},
$$

where $\overline{M A D}_{\text {ip }}$ is the isometric static population mean of the MAD at a particular target force level. $M A D_{\mathrm{t}}$ is a measure of the subject's tremor at a particular force level normalized by the target force and $M A D_{\mathrm{s}}$ is a measure of the subject's tremor at a particular force level compared to the population's tremor in the isometric case for the same force level. Note that the divisors of $M A D_{\mathrm{t}}$ and $M A D_{\mathrm{s}}$ are significantly different, so we should expect numerical values of these metrics to be significantly different. A value of zero for the metric $E_{\mathrm{t}}$ indicates that the subject can apply his/her mean force at the target force perfectly, whereas a value of zero for $E_{\mathrm{S}}$ implies that the subject can apply his/her mean force as the population would in the 
isometric case. Similarly, a value of zero for $M A D_{\mathrm{s}}$ implies that the subject has a $M A D$ similar to the $M A D$ of the population in the isometric case. A value of zero for $M A D_{\mathrm{t}}$ would indicate that there is no variation in the force applied by the subjects (i.e., no tremor in the applied force), which will never happen in practice. The four metrics were chosen to explore different aspects of force control ability. We would expect that, if a given trial was truly indistinguishable from the isometric case, then it should be indistinguishable using all four metrics. For the purpose of precise control of admittance-type devices, we believe $M A D_{\mathrm{t}}$ to be particularly important, as previously discussed.

To utilize the above metrics, we must first develop an understanding for the ways that the subjects apply forces isometrically, which provides a measure of force control ability when the subject is unaffected by the device in any way. Figure 6a shows the mean force in each trial $(\bar{F})$ for the isometric case across all subjects and trials, normalized by the target force. A value of 1 for $\bar{F} / F_{\mathrm{t}}$ would mean that we are able to perfectly maintain the target force. We see a tendency to apply forces higher than the target force at forces below approximately $2 \mathrm{~N}$ and to apply forces lower than the target force at forces higher than $2 \mathrm{~N}$. Figure $6 \mathrm{~b}$ shows the mean force in each trial $(\bar{F})$ for the isometric case across all subjects and trials, normalized by the isometric population mean $\left(\bar{F}_{\text {ip }}\right)$. This removes the effect of force level from the metric and results in a mean value of 1 for the isometric case irrespective of the target force level. We observe that the variability in mean force is relatively high at forces below $0.5 \mathrm{~N}$. MAD normalized by the target force is also found to be high at forces below $0.5 \mathrm{~N}$ (Fig. 7a). $\bar{F} / F_{\mathrm{t}}$ and $M A D / F_{\mathrm{t}}$ remain fairly constant for forces higher than $2 \mathrm{~N}$ (see Figs $6 \mathrm{a}$ and $7 \mathrm{a}$ ). Normalizing $M A D$ by the isometric population mean removes the effect of force level on the metric (Fig. 7b). Also, the variability in $M A D$ remains fairly constant after normalizing by $\overline{M A D}_{\text {ip }}$. The results reported in Figs $6 \mathrm{a}$ and $7 \mathrm{a}$ indicate that we have relatively poor force control at forces below $0.5 \mathrm{~N}$.

Once equipped with an understanding of the subjects' ability to maintain isometric forces, a fixed-effect ANOVA model was used to test the main effects of factors $V$ and $k$ on the four metrics (5)-(8). Figures 8-11 show the same experimental data set considered with respect to the metrics $E_{\mathrm{t}}, E_{\mathrm{s}}, M A D_{\mathrm{t}}$ and $M A D_{\mathrm{s}}$, respectively. Within each figure, four plots are included to show the data for a given metric represented in four different ways, each of which provides insight to the underlying behavior.

In Fig. 8 we can see that the metric $E_{\mathrm{t}}$, which is a measure of the subject's ability to maintain a constant force as compared to the target force, varies significantly with $V$, but depends much less on $k$ for the highest velocities. At the lowest velocities, $E_{\mathrm{t}}$ seems to increase with increasing $k$.

In Fig. 9, we can see that the metric $E_{\mathrm{s}}$, which is a measure of the subject's ability to maintain a constant target force as compared to the population's ability to maintain the same target force isometrically, shows a strong dependence on $k$. $E_{\mathrm{s}}$ is 


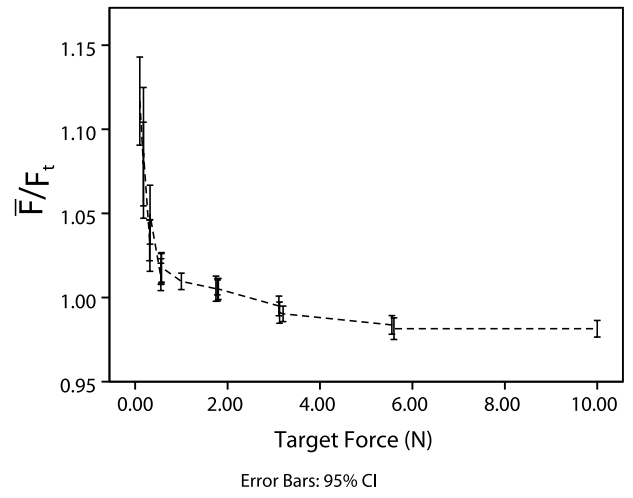

(a)

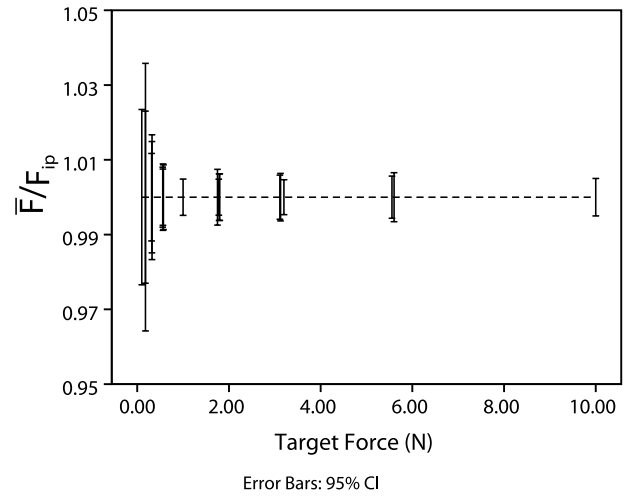

(b)

Figure 6. Mean force for the isometric case normalized by (a) target force and (b) isometric population mean, for all nine subjects combined.

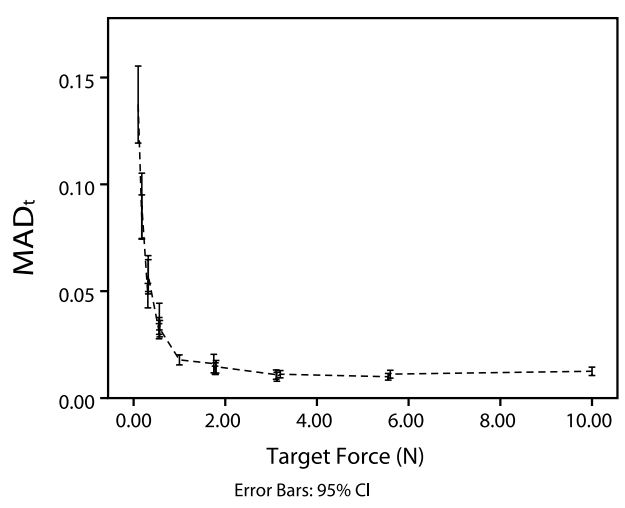

(a)

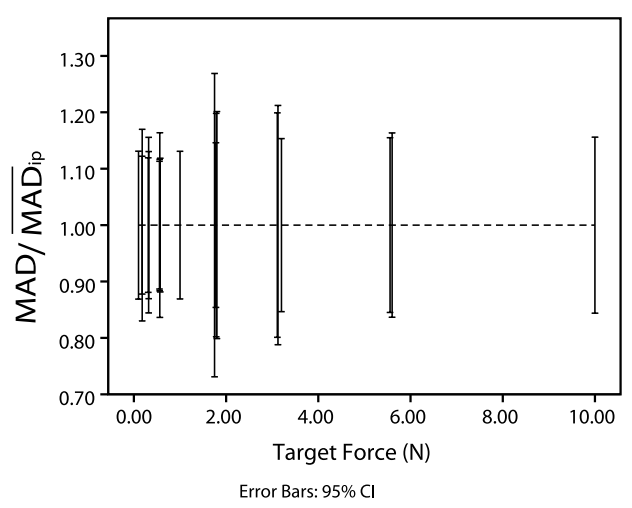

(b)

Figure 7. $M A D$ for the isometric case normalized by (a) target force (i.e., $M A D_{\mathrm{t}}$ ) and (b) isometric population mean of mean absolute deviations $\left(\overline{M A D}_{\mathrm{ip}}\right)$, for all nine subjects combined.

quite insensitive to both $V$ and the target force level at low values of $k$, and shows dependency as $k$ is increased.

In Fig. 10, we can see that $M A D_{\mathrm{t}}$, which is a measure of the subject's tremor at a particular force level normalized by the target force, depends on both $k$ and $V$, with $M A D_{\mathrm{t}}$ linearly proportional to $k$ for a constant value of $V$. Recall that $M A D_{\mathrm{t}}$ is essentially a measure of the input noise to our admittance-type device. We observe an increase in $M A D_{\mathrm{t}}$ with a decrease in $V$, as well as with a decrease in target force. We also see that, if the target force is held constant, then $M A D_{\mathrm{t}}$ seems to lose its dependence on $k$ and vary only with $V$.

In Fig. 11, we see the results for $M A D_{\mathrm{s}}$, which is a measure of the subject's tremor at a particular force level compared to the population's tremor in the isometric case for the same force level. The trends observed in this metric are not as 


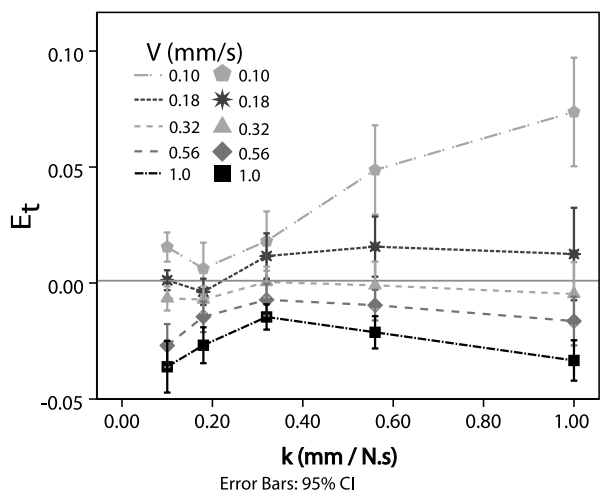

(a)

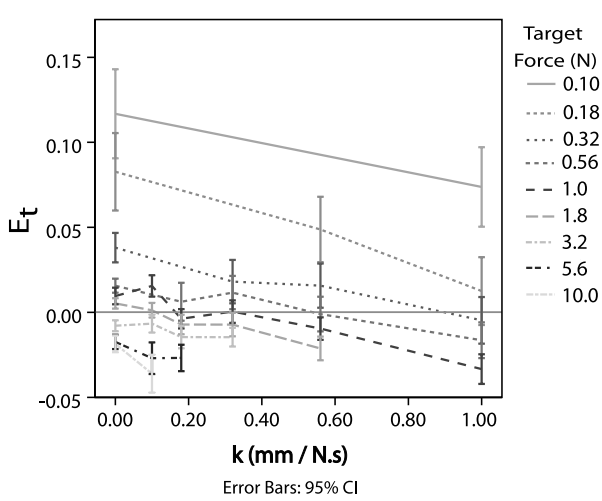

(c)

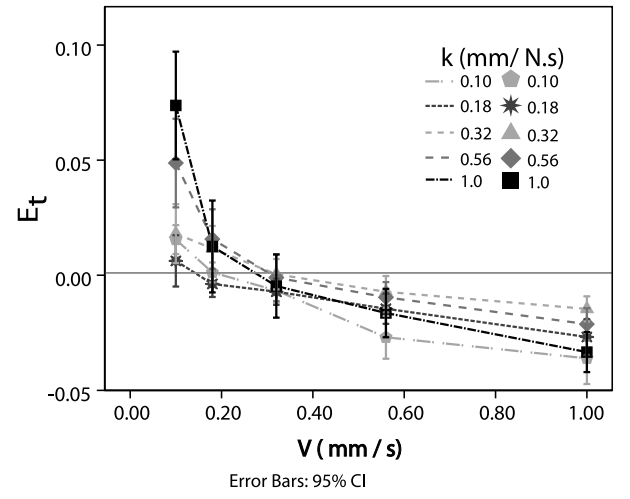

(b)

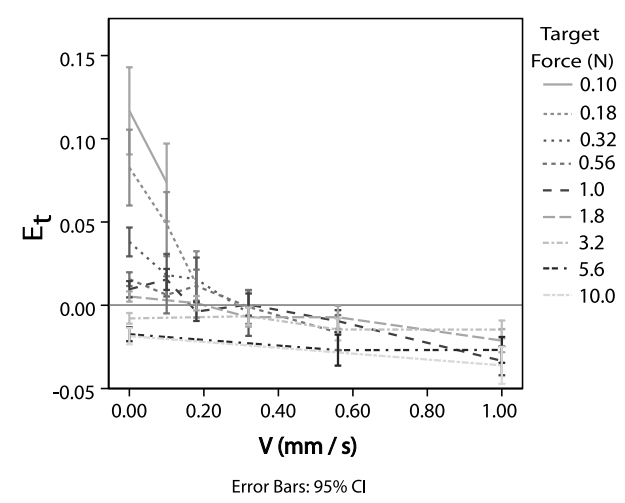

$(\mathrm{d})$

Figure 8. Experimental results for metric $E_{\mathrm{t}}$ for the low-speed experiment, for all nine subjects combined. $E_{\mathrm{t}}$ is a measure of the subject's ability to maintain a constant target force, as a fraction of the target force and is defined in (5).

pronounced as observed in the other three. The metric seems to depend on both $k$ and $V$.

ANOVA on the experiment indicates that both $V$ and $k$ have a main effect on the metrics $E_{\mathrm{t}}$ and $M A D_{\mathrm{t}}(p<0.01)$. The ability to accurately apply a target force, which corresponds to a low value of $E_{\mathrm{t}}$, seems to degrade at very low velocities and at high velocities (Figs 8 and A1). Also, the user's tremor normalized by the target force, $M A D_{\mathrm{t}}$, seems to improve with increasing $V$ (Fig. A3). On closer analysis, we find that both $E_{\mathrm{t}}$ and $M A D_{\mathrm{t}}$ have a strong dependence on the force level. Figures $8 \mathrm{~d}$ and $10 \mathrm{~d}$ show an insensitivity to force and movement for the metrics at forces higher than $2 \mathrm{~N}$. For target forces of 3.2, 5.6 and $10 \mathrm{~N}$ the values of metrics $E_{\mathrm{t}}$ and $M A D_{\mathrm{t}}$ remain approximately constant for all values of $V$.

ANOVA for metrics $E_{\mathrm{s}}$ and $M A D_{\mathrm{s}}$, which quantify subjects' ability to accurately apply a target force relative to the population's performance in the isometric appli- 


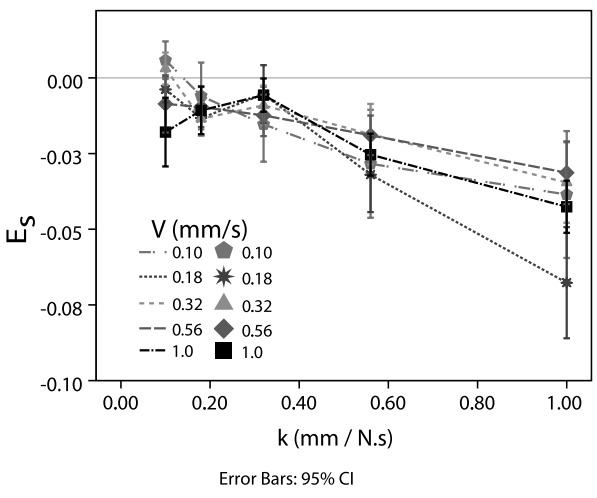

(a)

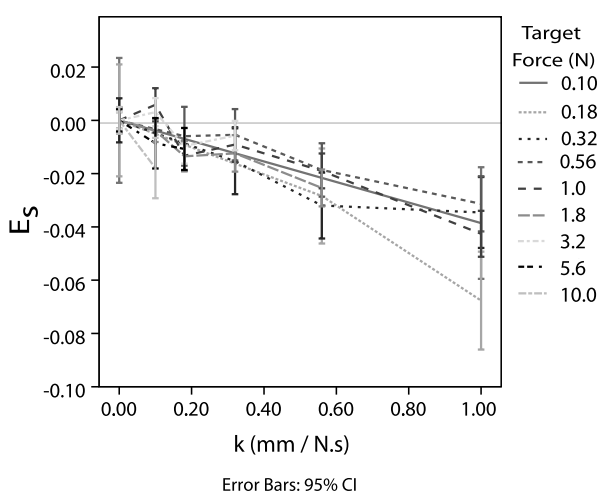

(c)

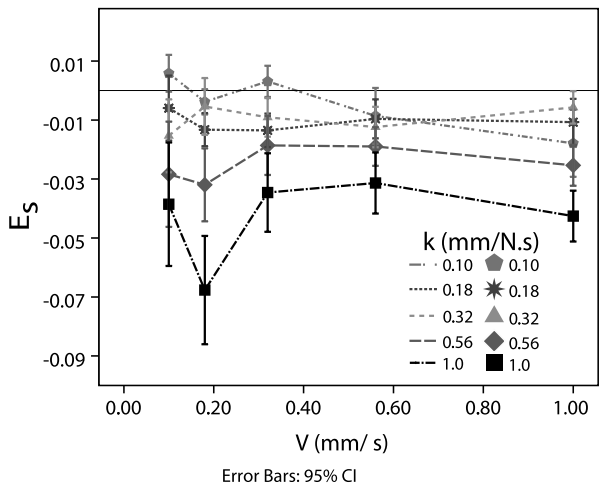

(b)

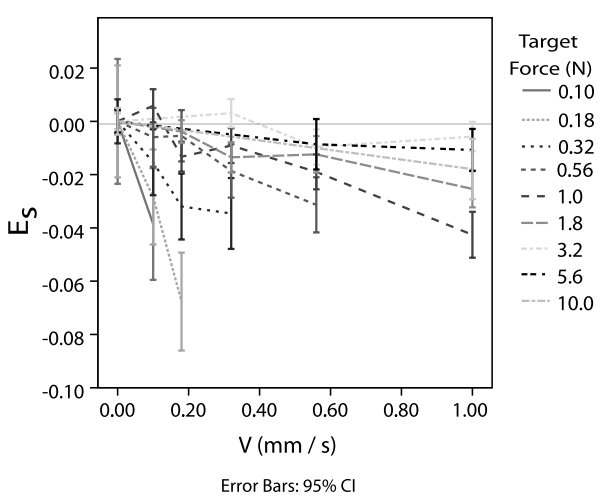

(d)

Figure 9. Experimental results for metric $E_{\mathrm{S}}$ for the low-speed experiment, for all nine subjects combined. $E_{\mathrm{S}}$ is a measure of the subject's ability to maintain a constant target force as compared to the population's ability to maintain the same target force and is defined in (6).

cation of the same target force, show that both $V$ and $k$ have a main effect on the metrics $(p<0.01)$. As these metrics have been normalized by the isometric population means at each respective target force, the effect of force levels on these metrics is not as apparent as it was with $E_{\mathrm{t}}$ and $M A D_{\mathrm{t}}$. The results in Fig. 9 show that $E_{\mathrm{s}}$ degrades rapidly with an increase in $k$ and remains approximately constant for different values of $V$. At high values of $k$, a slight improvement in performance is seen with increasing $V$ (Fig. A2b). For values of $k$ below $0.32 \mathrm{~mm} /(\mathrm{N} \cdot \mathrm{s})$ performance is within $1 \%$ of the isometric case. At $k=0.1 \mathrm{~mm} /(\mathrm{N} \cdot \mathrm{s})$ the value for $E_{\mathrm{s}}$ is distributed around zero, indicating that $E_{\mathrm{s}}$ is similar to the isometric case. Although $M A D_{\mathrm{s}}$ seems to have less structure at first, it can be seen that for $k=0.1 \mathrm{~mm} /(\mathrm{N} \cdot \mathrm{s})$, $M A D_{\mathrm{s}}$ is similar to the isometric case (Fig. $11 \mathrm{~b}$ ). $M A D_{\mathrm{s}}$ seems to be relatively high for all other values of $V$ and $k$. 


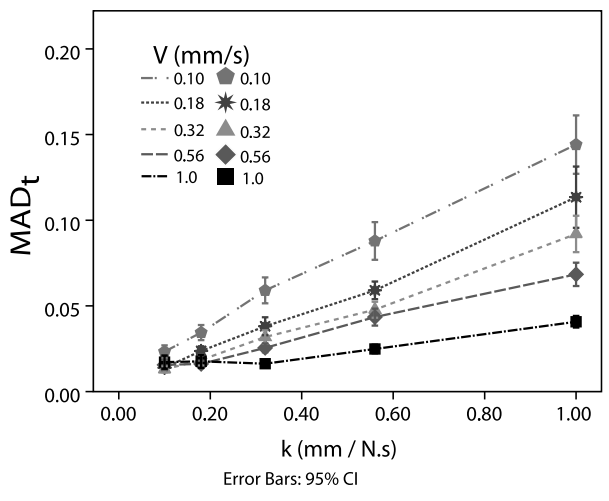

(a)

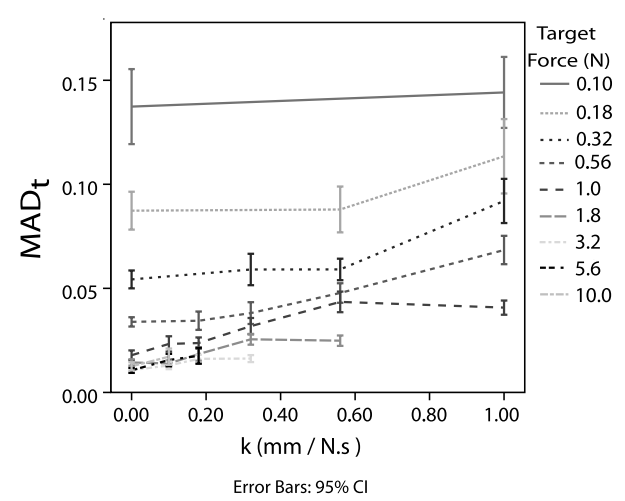

(c)

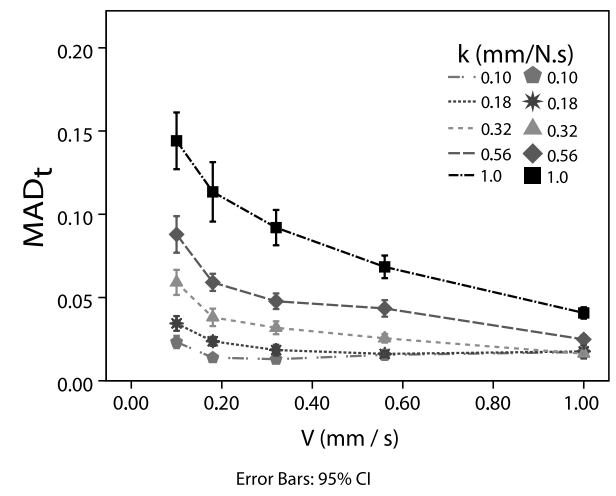

(b)

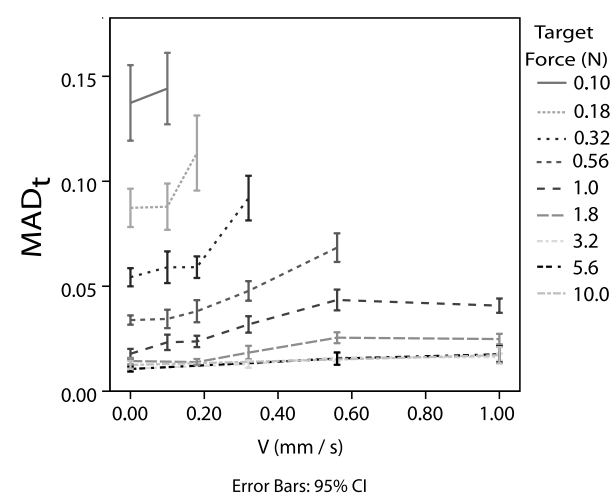

(d)

Figure 10. Experimental results for metric $M A D_{\mathrm{t}}$ for the low-speed experiment, for all nine subjects combined. $M A D_{\mathrm{t}}$ is a measure of the subject's tremor at a particular force level normalized by the target force and is defined in (7).

The partial effect sizes for the ANOVA tests performed are given in Table 2. The partial effect size gives the proportion of variance in the metric accounted for by a particular factor: $\eta_{k}^{2}$ gives the amount of variance in the metric accounted for by $k$ and $\eta_{V}^{2}$ gives the amount of variance in the metric accounted for by $V . \eta_{V}^{2}$ is higher than $\eta_{k}^{2}$ in both of the experiments for the metric $E_{\mathrm{t}} . \eta_{k}^{2}$ is higher than $\eta_{V}^{2}$ for metrics $M A D_{\mathrm{t}}$ and $E_{\mathrm{s}}$. An examination of the partial effect sizes supports what was observed in Figs 8-11. One thing that is clear is that, for most of the metrics (excluding $M A D_{\mathrm{t}}$ ) the variance between individual trials (and likely between subjects) is large compared to the variance that is explained by $k$ and $V$.

\section{Discussion}

From our statistical analysis it can be seen that both admittance gain $(k)$ and velocity $(V)$ have an effect on force control precision. From analysis of results presented in 


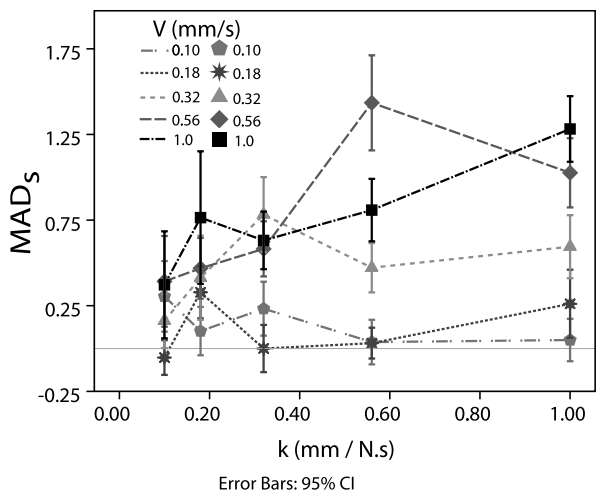

(a)

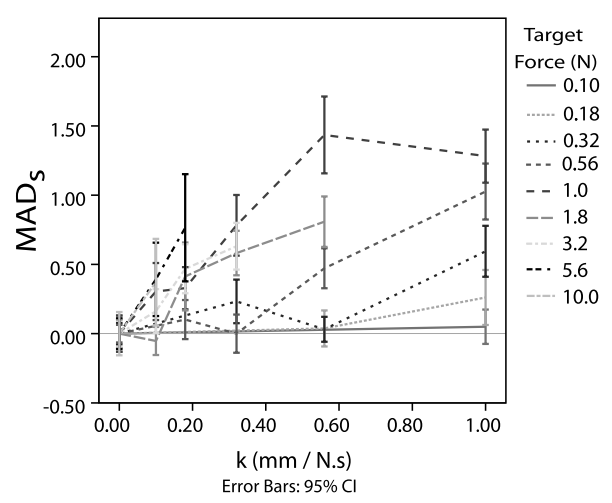

(c)

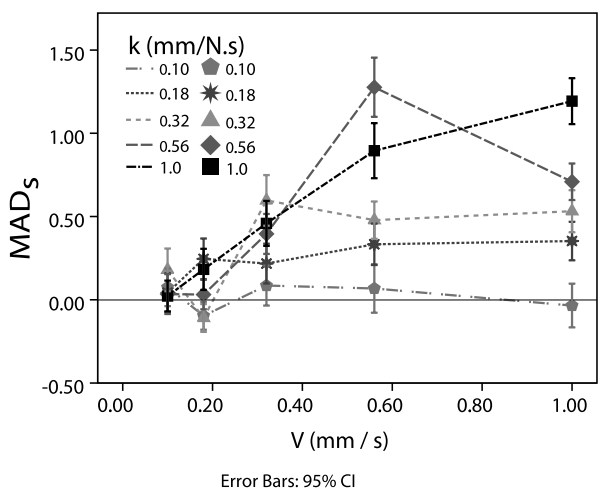

(b)

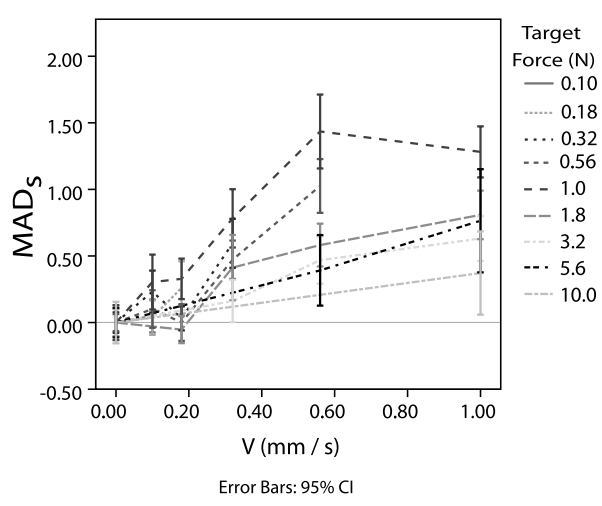

(d)

Figure 11. Experimental results for metric $M A D_{\mathrm{s}}$ for the low-speed experiment, for all nine subjects combined. $M A D_{\mathrm{s}}$ is a measure of the subject's tremor at particular force level compared to the population's tremor in the isometric case for the same force level and is defined in (8).

Table 2.

Partial effect sizes for ANOVA tests, with respect to admittance gain $\left(\eta_{k}^{2}\right)$ and velocity $\left(\eta_{V}^{2}\right)$, for the low-speed experiment

\begin{tabular}{lllll}
\hline & $E_{\mathrm{t}}$ & $E_{\mathrm{s}}$ & $M A D_{\mathrm{t}}$ & $M A D_{\mathrm{s}}$ \\
\hline$\eta_{k}^{2}$ & 0.03 & 0.10 & 0.49 & 0.03 \\
$\eta_{V}^{2}$ & 0.17 & 0.007 & 0.24 & 0.10 \\
\hline
\end{tabular}

this paper, it appears that the ability to control applied force degrades rapidly as we increase $k$ for the general population. Wu et al. [8] concluded that only velocity determines human force control precision during admittance control. In this paper we 
have provided compelling evidence that, at least in the velocity range considered here, both velocity and admittance gain have an effect on force control precision and that admittance gain has the most pronounced effect. Not all of the tests for force control performed in Ref. [8] were conducted using the proportional-velocity control law (1). This could explain the difference in results between the two studies. Additionally, their experimental procedure involved rapid accelerations, which do not typically appear in actual admittance-controlled devices, which might have affected the outcome by either providing cueing or masking to the subjects.

The metrics $E_{\mathrm{s}}$ and $M A D_{\mathrm{s}}$ show that we have performance similar to the isometric case at $k=0.1 \mathrm{~mm} /(\mathrm{N} \cdot \mathrm{s})$. At higher values of $k$, force control precision steadily decreases with increasing $k$, as seen in Figs 9 and 11. We observe that at $k=0.1 \mathrm{~mm} /(\mathrm{N} \cdot \mathrm{s})$ control precision remains constant for different values of $V$, whereas at $V=0.1 \mathrm{~mm} / \mathrm{s}$ control precision changes with varying $k$ (Figs $8 \mathrm{~b}, 9 \mathrm{~b}$, $10 \mathrm{~b}$ and $11 \mathrm{~b}$ ). Reduced performance is observed in the metric $E_{\mathrm{s}}$ at $V=0.18 \mathrm{~mm} / \mathrm{s}$ in Fig. 9b. The target force at this point is less than $0.5 \mathrm{~N}$ and what we are observing could be an effect of the high variability of force at such a low force level. Thus, at $k=0.1 \mathrm{~mm} /(\mathrm{N} \cdot \mathrm{s})$ it is like we are performing a quasistatic task and performance seems to be unaffected by movement.

For constant force, $k$ and $V$ are linearly coupled through control law (1). Force level seems to be a strong effect on metrics $E_{\mathrm{t}}$ and $M A D_{\mathrm{t}}$ (Figs $8 \mathrm{c}$ and 10c). We see a strong dependence on $V$ at low velocities. Low $V$ means low force and we know that our force control ability is poor at low forces (below $0.5 \mathrm{~N}$, see Fig. 6). The high partial effect size for $V$ on metric $E_{\mathrm{t}}$ could be an effect of low forces encountered at low velocities, since the ANOVA does not explicitly test for a main effect of $F$, as the experiment was not designed to be balanced in $F$.

Based on the control law (1), we could also conclude that poor force control at high $k$ and low $V$ is actually a measurement of poor force control at low values of applied force $F$. Although we normalized the metrics by the static case at each force value, in an attempt to control for the effect of changing the applied force level, it may be the case that when a subject is pushing harder and consequently has a stiffer finger, the subject is more robust to movement of the system. Based on the analysis of the data collected, human force control performance is best at low admittance gains. To obtain precise force control, $k$ should be kept as low as $0.1 \mathrm{~mm} /(\mathrm{N} \cdot \mathrm{s})$ if possible. However, this will require the application of large forces over sustained periods to generate useful velocities, which could increase operator fatigue, which in turn could result in loss of force control precision.

Finally, our results apply directly to force control on admittance-type devices, but it is not clear from this experiment how these results will extend to precise trajectory control of admittance-type devices. However, we do have some indication. Recall that $M A D_{\mathrm{t}}$ is a measure of normalized input noise to our system. $M A D_{\mathrm{t}}$ varies linearly with $k$ and we find $M A D_{\mathrm{t}}$ is more sensitive to changes in $k$ as velocity decreases, indicating that $k$ is a better predictor of force control precision than $V$ at low velocities (and target force is a better predictor than both $k$ and $V$ ). Consider 
a human operator controlling an admittance-type micromanipulation system under guidance of an optical microscope. If the human attempts to do a slow-moving (quasistatic) precise positioning task, the micromanipulator's tremor that is visually observed (relative to the intentional movements) will essentially be $M A D_{\mathrm{t}}$. In any positioning task, we must reduce the speed to zero and there are two ways to accomplish this: push softer or reduce $k$. We have seen that humans have poor force control at low forces (i.e., they have high $M A D_{\mathrm{t}}$ at less than $0.5 \mathrm{~N}$ ), which suggests the possible use of a deadband to restrict any motion at very low force. However, it may also be possible to change to a lower fixed $k$ value for low velocities during high-precision tasks or to implement a nonlinear control law (e.g., the quadratic law $V=k F^{2}$ ) to attenuate the effect of tremor at low velocities. Both a deadband and a quadratic controller are being implemented on the University of Utah Active Handrest [4].

\section{Conclusions}

In this paper, we have studied the effects of velocity $(V)$, admittance gain $(k)$ and force $(F)$ on human force control ability when using admittance-type devices, which have applied force as their input signal. We believe that humans feel most comfortable interacting with devices that have a dissipative dynamic behavior, and that there exists a range of forces and velocities within which humans optimally interact with admittance-type devices. We found that the nominal force level is the most important factor in determining force control precision and human force control ability is poor at very low forces (below $0.5 \mathrm{~N}$ ). To maintain a high level of control over the movement of the device, $k$ should be chosen such that meaningful interactions require nominal forces larger than $0.5 \mathrm{~N}$. This can be accomplished by the use of a deadband and/or by varying the effective admittance value to attenuate the effects of tremor at low force inputs. Once the effect of force was accounted for, the admittance gain $(k)$ was found to be the second-most important factor in determining force control precision. At $k=0.1 \mathrm{~mm} /(\mathrm{N} \cdot \mathrm{s})$, force control precision was found to be similar to the isometric case (i.e., when the device is stationary). For this admittance value, the human force input can be regarded as an exogenous system input, rather than treating the human as a part of the closed-loop feedback system. This can be used to make higher-performance, less-conservative controller designs. Even with a small increase in $k$, force control precision degrades measurably, including during very slow movements that are barely perceived as moving. For the highest level of precision, low admittance gains are recommended, but it could result in operator fatigue due to application of large forces. For optimal performance, a tradeoff has to be reached between force control precision and operator comfort. 


\section{References}

1. R. J. Adams and B. Hannaford, Stable haptic interaction with virtual environments, IEEE Trans. Robotics Automat. 15, 465-474 (1999).

2. R. Taylor, P. Jensen, L. Whitcomb, A. Barnes, R. Kumar, D. Stoianovici, P. Gupta, Z. Wang, E. deJuan and L. Kavoussi, Steady-hand robotic system for microsurgical augmentation, Int. J. Robotics Res. 18, 1201-1210 (1999).

3. B. Mitchell, M. John Koo, I. Iordachita, P. Kazanzides, A. Kapoor, M. James Hunda, G. Hager and R. Taylor, Development and application of a new steady-hand manipulator for retinal surger, in: Proc. IEEE Int. Conf. Robotics and Automation, Rome, pp. 623-629 (2007).

4. M. A. Fehlberg, B. T. Gleeson, L. C. Leishman and W. R. Provancher, Active handrest for precision manipulation and ergonomic support, in: Proc. Symp. on Haptic Interfaces for Virtual Environments and Teleoperator Systems, Waltham, MA, pp. 489-496 (2010).

5. M. A. Srinivasan and J.-S. Chen, Human performance in controlling normal forces of contact with rigid objects, in: Advances in Robotics, Mechatronics, and Haptic Interfaces, DSC Volume 49, pp. 119-125. ASME, New Orleans, LA (1993).

6. S. Allin, Y. Matsuoka and R. Klatzky, Measuring just noticeable differences for haptic force feedback: implications for rehabilitation, in: Proc. Symp. on Haptic Interfaces for Virtual Environments and Teleoperator Systems, Orlando, FL, pp. 299-302 (2002).

7. S. J. Lederman, R. D. Howe, R. L. Klatzky and C. Hamilton, Force variability during surface contact with bare finger or rigid probe, in: Proc. Symp. on Haptic Interfaces for Virtual Environments and Teleoperator Systems, Chicago, IL, pp. 154-160 (2004).

8. M. Wu, J. J. Abbott and A. M. Okamura, Effect of velocity on human force control, in: Proc. Joint Eurohaptics Conf. and Symp. on Haptic Interfaces for Virtual Environment and Teleoperator Systems, Pisa, pp. 73-79 (2005).

9. L. A. Jones, Visual and haptic feedback in the control of force, Exp. Brain Res. 130, 269-272 (2000).

10. CHAI 3D: The Open Source Haptics Project, http://www.chai3d.org/.

11. M. Nambi, W. R. Provancher and J. J. Abbott, Revisiting the effect of velocity on human force control, Lecture Notes Comp. Sci. 6191, 144-151 (2010).

\section{Appendix}

Data for the high-speed experiment is shown in this Appendix. Figures A1-A4 show a single experimental data set considered, respectively, for the metrics $E_{\mathrm{t}}, E_{\mathrm{s}}$, $M A D_{\mathrm{t}}$ and $M A D_{\mathrm{s}}$. Within each figure, two plots are included to show the data for a given metric represented in two different ways, each of which provides insight to the underlying behavior. Table A1 shows the partial effect sizes for ANOVA tests. This high-speed experiment was used to inform the design of the low-speed experiment, which was discussed in detail in Sections 3 and 4. The high-speed experiment was also conducted as described in Section 2. 
Table A1.

Partial effect sizes for ANOVA tests, with respect to admittance gain $\left(\eta_{k}^{2}\right)$ and velocity $\left(\eta_{V}^{2}\right)$, for the high-speed experiment

\begin{tabular}{lllll}
\hline & $E_{\mathrm{t}}$ & $E_{\mathrm{s}}$ & $M A D_{\mathrm{t}}$ & $M A D_{\mathrm{s}}$ \\
\hline$\eta_{k}^{2}$ & 0.013 & 0.392 & 0.061 & 0.017 \\
$\eta_{V}^{2}$ & 0.24 & 0.052 & 0.055 & 0.029 \\
\hline
\end{tabular}

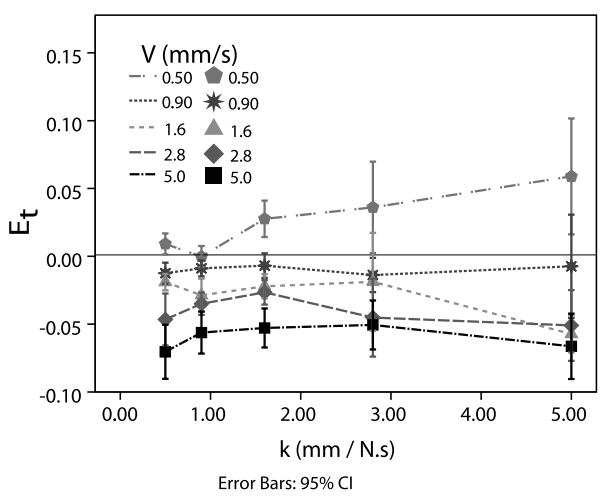

(a)

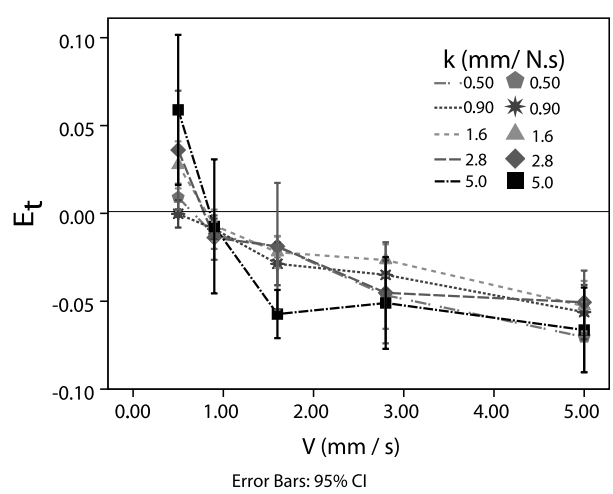

(b)

Figure A1. Experimental results for metric $E_{\mathrm{t}}$ for the high-speed experiment, for all nine subjects combined. $E_{\mathrm{t}}$ is a measure of the subject's ability to maintain a constant target force, as a fraction of the target force and is defined in (5).

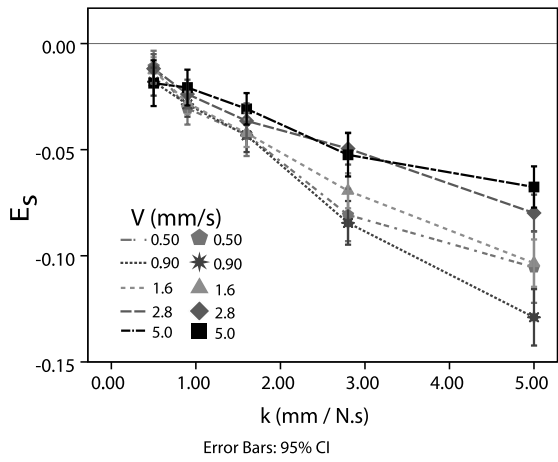

(a)

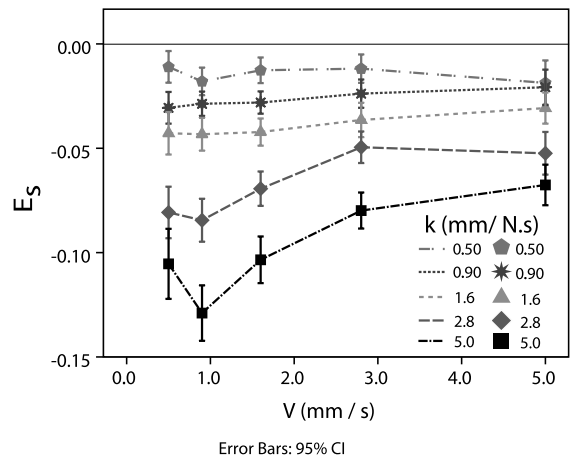

(b)

Figure A2. Experimental results for metric $E_{\mathrm{S}}$ for the high-speed experiment, for all nine subjects combined. $E_{\mathrm{S}}$ is a measure of the subject's ability to maintain a constant target force as compared to the population's ability to maintain the same target force and is defined in (6). 


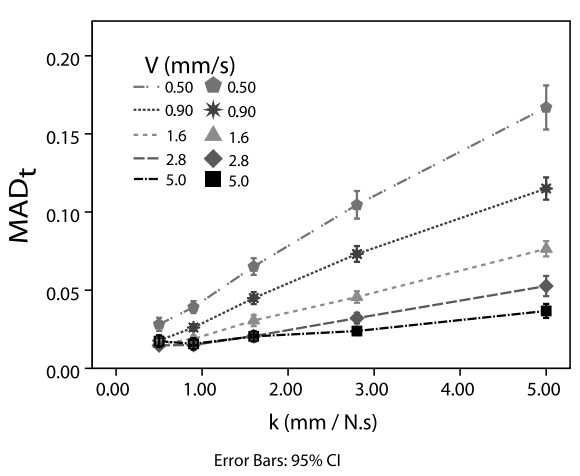

(a)

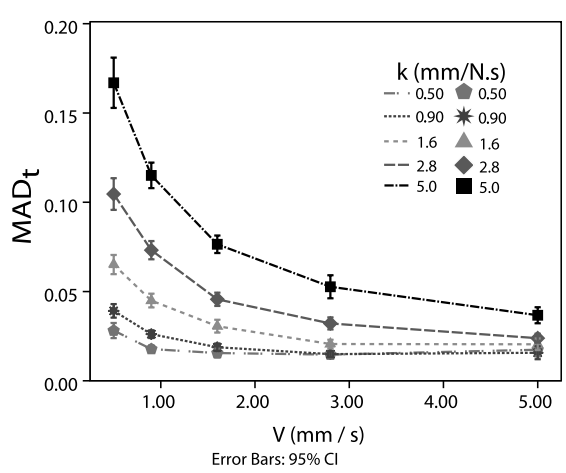

(b)

Figure A3. Experimental results for metric $M A D_{\mathrm{t}}$ for the high-speed experiment, for all nine subjects combined. $M A D_{\mathrm{t}}$ is a measure of the subject's tremor at a particular force level normalized by the target force and is defined in (7).

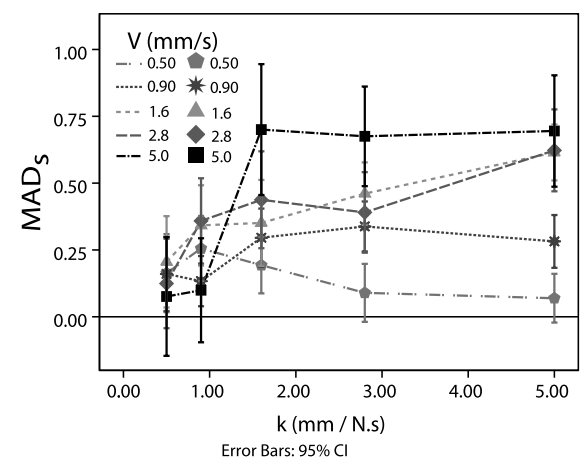

(a)

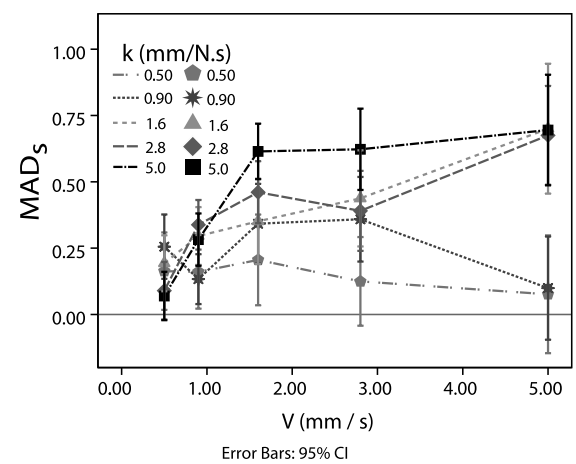

(b)

Figure A4. Experimental results for metric $M A D_{\mathrm{s}}$ for the high-speed experiment, for all nine subjects combined. $M A D_{\mathrm{S}}$ is a measure of the subject's tremor at particular force level compared to the population's tremor in the isometric case for the same force level and is defined in (8).

\section{About the Authors}

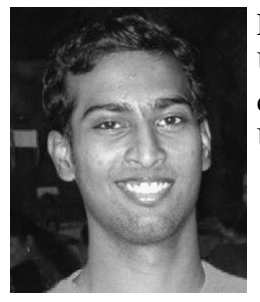

Manikantan Nambi received the BE degree in Mechanical Engineering from the University of Mumbai, India, in 2008. He is currently working towards a $\mathrm{PhD}$ degree in the Telerobotics Laboratory, Department of Mechanical Engineering, University of Utah, where he is investigating micromanipulation systems. 


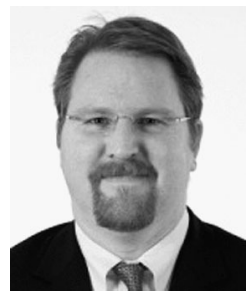

William R. Provancher earned a BS in Mechanical Engineering and an MS in Materials Science and Engineering, both from the University of Michigan. He earned a PhD degree in Mechanical Engineering at Stanford University, working in the area of haptics and tactile sensing and feedback. His postdoctoral research involved the design of bioinspired climbing robots. He is currently an Assistant Professor in the Department of Mechanical Engineering at the University of Utah. He teaches courses in the areas of mechanical design, mechatronics and haptics. His active areas of research include haptics and the design of novel climbing robots. He received a Best Paper Award at the 2009 World Haptics Conference for his work on tactile feedback for the communication of direction.

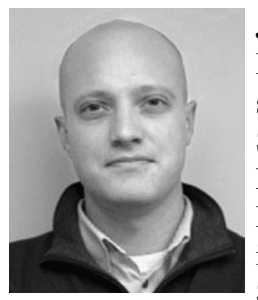

Jake J. Abbott received the PhD degree in Mechanical Engineering from Johns Hopkins University, Baltimore, MD, in 2005. He became a Postdoctoral Research Associate at the Institute of Robotics and Intelligent Systems, ETH Zurich, Switzerland, in 2005, and an Assistant Professor at the University of Utah, Salt Lake City, UT, in 2008. He is currently the Head of the Telerobotics Laboratory, Department of Mechanical Engineering, University of Utah, where his research involves medical and microscale telerobotics. He is a recipient of the National Science Foundation CAREER Award, and he received the Best Manipulation Paper Award at the 2010 IEEE International Conference on Robotics and Automation. 